\title{
Further experiments on collisional tribocharging of cosmic grains
}

\author{
T. Poppe and R. Schräpler
}

\author{
Institut für Geophysik und extraterrestrische Physik, Technische Universität Braunschweig, Mendelssohnstraße 3, \\ 38106 Braunschweig, Germany \\ e-mail: t.poppe@tu-bs.de
}

Received 8 November 2004 / Accepted 8 March 2005

\begin{abstract}
We experimentally investigated tribocharging in bouncing dust grain collisions under conditions which are relevant for the early solar nebula. We used silica, diamond, graphite, and silicon carbide grains and $\mathrm{cm}$-sized targets of aluminum, iron, and graphite. We varied particle size around $1 \mu \mathrm{m}$, collision velocity between 38 to $106 \mathrm{~m} \mathrm{~s}^{-1}$, and target temperature between $240 \mathrm{~K}$ and $360 \mathrm{~K}$. Generally, the grains acquire something in the order of hundreds or thousands of negative elementary charges, which is at the upper end of the range previously discussed in astrophysical research. Mainly, a temperature difference caused by the dissipation of collisional energy or by initial temperatures determines the amount of charge transfer. It suggests a thermally diffusive charge transfer process of negative charge carriers. Nevertheless, also other processes of secondary importance were effective. Material-dependent contact charging is ruled out as the dominant charge transfer process in our experiments. For most particle-target combinations, the charge transfer can be described by an empirical function of collision energy which we propose for future astrophysical applications, especially for applications on the sticking efficiency in the preplanetary dust aggregation process, on the particle motion in the solar nebula, and on the possibility of nebular lightning.
\end{abstract}

Key words. solar system: formation - methods: laboratory - dust, extinction

\section{Introduction}

When solid grains collide, an electrical charge transfer can occur due to tribocharging. This process was regarded as important for numerous applications in various disciplines and was also relevant for physical processes which formed the solar system.

In the solar nebula, the planet formation process began with a dust aggregation of grains of primarily micrometer size, which were embedded in dilute gas, collided, and then stuck (e.g. Weidenschilling \& Cuzzi 1993). For this process, the sticking efficiency in dust grain collisions is an important parameter. In experimental work with the aim of investigating sticking probabilities (Poppe et al. 2000a,b), it became obvious that triboelectrification can contribute to the sticking efficiency. Grains which mechanically bounced off were reattracted by collision-induced electrostatic forces and finally stuck.

Also in the solar nebula, lightning-like gas discharges could have occurred. Steps in this process are that particles embedded in an almost neutral gas collided and charged each other. Then they were separated and concentrated by gas flow patterns of the solar nebula such that electric fields built up to compete with particle discharging due to a weak ionisation of the gas, e.g. resulting from the decay of radioactive elements. If the field is strong enough an electrical breakthrough will occur and equilibrate the electrical charges by "lightning". The discussion dates back to Whipple (1966) and is linked to meteoritic chondrule formation. Chondrules are spheroid stony meteoritic components of typically $0.1-1 \mathrm{~mm}$ diameter which underwent a transient heating process that melted them; the heating process, whether lightning or not, is believed to have taken place outside those meteorites in which the chondrules are found today (e.g. Hewins 1996). Important work on the possibility of lightning has been done by Morfill et al. (1993), Love et al. (1995), Gibbard et al. (1997), Pilipp et al. (1998), and Desch \& Cuzzi (2000).

Furthermore, the dynamics of dust populations is influenced by electric and magnetic fields when the dust is charged. With respect to particle charge-to-mass ratios which were even lower than most measured in this work, Poppe et al. (2000b) already pointed out that forces caused by magnetic fields could be stronger than aerodynamical forces in the early solar nebula.

For the above-mentioned applications, solar nebula conditions are important, and particle sizes and collision velocities must be considered. Typical particle sizes are in the micrometer range as supported by meteoritic studies on preplanetary dust before particle accretion (Kerridge 1993). Evidence based on the investigation of interplanetary dust particles (IDPs) often of cometary origin (Rietmeijer 2002; Brownlee 1985; Jessberger et al. 2001; Sandford 1996) makes a size range around $1 \mu \mathrm{m}$ appear typical for preplanetary dust. Rietmeijer (2002) measured sizes of "granular units" in an IDP and found a range of $64 \mathrm{~nm}$ to $7580 \mathrm{~nm}$ with a mean value of $585 \mathrm{~nm}$. Jessberger et al. (2001) mentioned $0.3 \mu \mathrm{m}$ as typical size of the original grains. The collision velocities in the solar nebula range up to $50 \mathrm{~m} \mathrm{~s}^{-1}$, 
which is believed to be characteristic for collisions of micronsized grains with large objects of $\mathrm{m}$ size (Weidenschilling \& Cuzzi 1993), if only inward drift and sedimentation are treated. Eddies may cause even higher collision velocities. The turbulent velocity was between 20 and $200 \mathrm{~m} \mathrm{~s}^{-1}$ at $1 \mathrm{AU}$ (Cuzzi et al. 1993). Assuming collisions of micron-sized particles that are fully coupled to the turbulent nebula gas with large objects that are decoupled from the turbulent motions, the collision velocities fall in the same range (Schräpler \& Henning 2004).

It was our intention to investigate tribocharging by experiments on the charge transfer in bouncing grain-target collisions with cosmic dust analogues. We introduce tribocharging, then describe the analogous materials and report measurements of collisional charge transfer with varying conditions: particle samples, target materials, and collision energy. The results indicated that a collision-induced temperature gradient is important for grain charging. Consequently, we then conducted further experiments varying the target temperature. Finally, we discuss the results with regard to the physical nature of tribocharging and to astrophysical applications.

\section{Tribocharging: An overview}

Tribocharging is one of the oldest problems in physical science, which however, still lacks an established theory. It is a collective term for numerous processes of charge transfer between solids or liquids. Possibly the most comprehensive treatment is found in Harper (1967).

\subsection{Contact charging}

Probably the widest-spread physical idea is the one of contact charging for which the work functions of the contacting materials are important. The idea is that a difference in the work function makes it energetically favorable for electrons to change the objects in contact, and a charge imbalance remains after separation leaving the objects electrified. As a consequence of this hypothesis, triboelectric series predict the charging sign of an object according to the material it is made of. However there is neither a reason why contact charging should be the most important process nor why it should be regarded as well-understood. Problems are firstly that triboelectric series have numerous contradictions (Harper 1967; Vercoulen 1995). Secondly, the work function of insulators are, at least, hard to determine. To overcome this problem, many authors (e.g. Lowell \& Rose-Innes 1980; Sternovsky et al. 2002) introduced "effective work functions" fitted to the results of tribocharging experiments. One objection to this is that it would theoretically explain a wide variety of electrification processes in the frame of contact charging, and the more or less arbitrary effective work functions assigned to insulators evade any verification by other than tribocharging experiments. In contrast to insulators, the work functions of metals are well known but the application is difficult because the separation process of conducting materials allows a reflow of charges and thus plays a major role in electrification, as well as the fact that due to conductance the charge does not accumulate at the surface (e.g. Lowell \& Rose-Innes 1980).

\subsection{Other processes in physical literature}

The role of motion and friction in contact charging is as intricate as their role in tribocharging in general. It is known from the contact with liquid mercury that charge transfer can occur without friction (Lowell 1986; Harper 1967), and effort has been made to eliminate relative motion in tribocharging experiments (Lowell 1986). If motion is involved, a subtle distinction between "sliding" and "rubbing" is made, the first involving gentle motion, the latter harder motion, whether with respect to contact pressure or velocity (Harper 1967, and references therein; Lowell \& Rose-Innes 1980). Sliding is believed to increase contact charging by multiplying the contact points while rubbing itself is a cause for charge transfer, as can be seen from one of few well-established facts that identical objects like sticks which are rubbed against each other can charge upon contact (Harper 1967; Lowell \& Rose-Innes 1980). Even the sign can change depending on the way of rubbing, i.e. on the role as rubbing or as rubbed object, or as "bow" or as "string". One among other hypotheses is that "hot spots" (Harper 1967) are important where charge carriers are more mobile and where motion-induced temperature differences cause a diffusive transfer of charge carriers. Thus temperature gradients in general must be regarded as important for charge transfer. Bowles's (1961) experiments on collisions of macroscopic bodies with different temperatures showed a diffusive electrification which involved positive charge carriers, and aside from the diffusive process at least one further electrification process had been effective. Lowell and Rose-Innes (1980) related former descriptions and the experimental results (Bowles 1961) of temperature-dependent charge carrier transfer to the transfer of ions which they present as explanation of charge transfer between like surfaces at temperature differences. Beyond the electrification processes listed above, also the transfer of charged impurities or charged rubbed-off bulk material is discussed in the afore mentioned literature on tribocharging.

\subsection{Charge transfer in particle collisions}

There is also literature on charge transfer in particle collisions which treats aerosol particles colliding with macroscopic objects. Many authors treat their results within the hypothesis of contact charging (Matsuyama \& Yamamoto 1989; John et al. 1980; Kacprzyk \& Gajewski 2001; Masuda et al. 1976; Cheng \& Soo 1970), although the explanation of results is not fully satisfying. The application of former tribocharging experiments with small particles is restricted by the experimental parameters because mostly the velocities were smaller, the particles larger than desirable, the materials were hardly relevant for our applications, and the role of surrounding gas is an open issue as is suggested by a particle measurement technique which involves ions adhering to charged particles. It worked such that charge of the ions is detected when transferred to to the target upon particle impact (Prochazka 1966). 

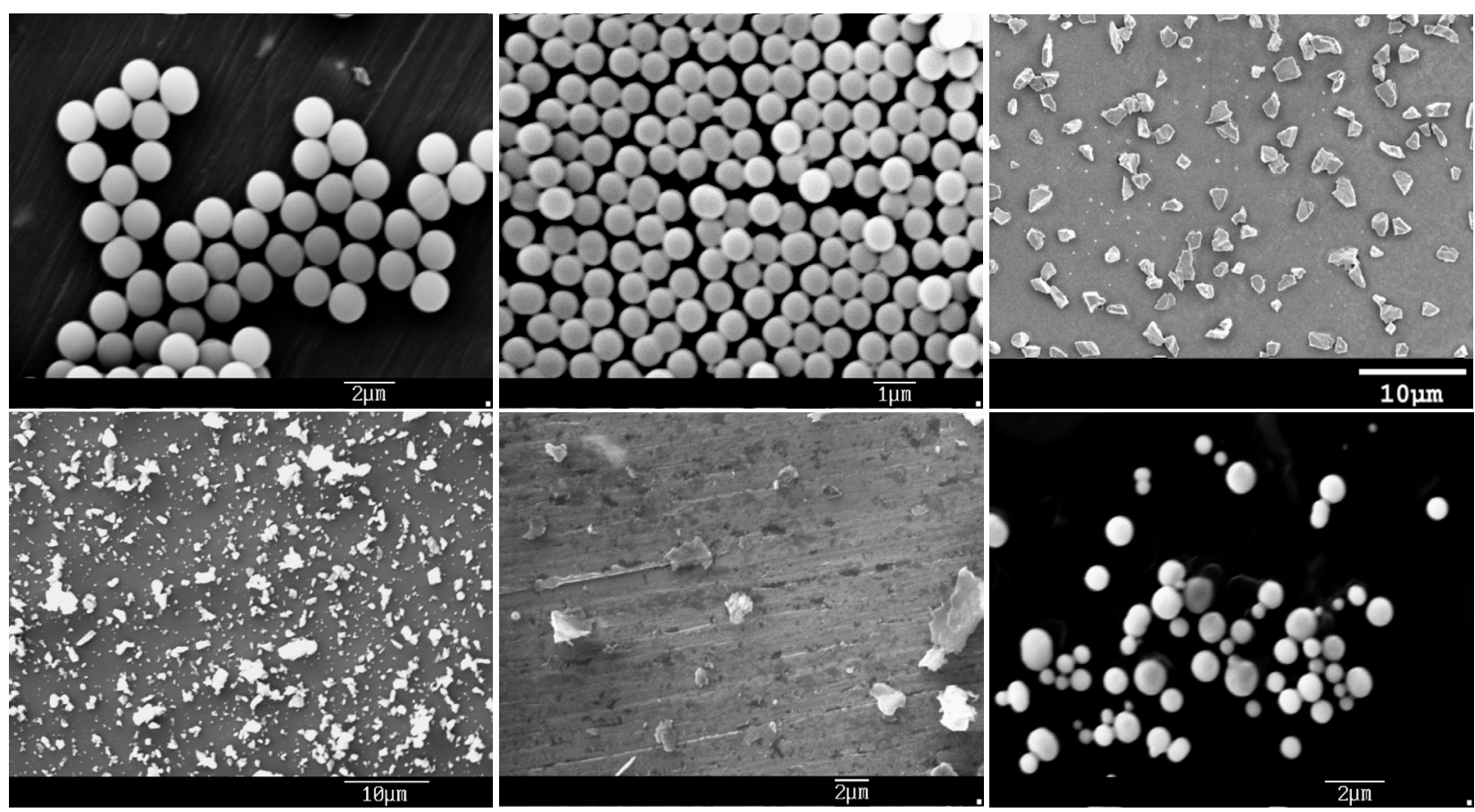

Fig. 1. SEM images of the dust samples. From left to right, from top to bottom: Large silica spheres, small silica spheres, diamond grains, $\mathrm{SiC}$ grains, graphite grains, and iron spheres.

\subsection{Treatment in astrophysical literature}

More tribocharging processes than discussed so far were treated in astrophysical literature on the possibility of nebular lightning. The processes of thunderstorm electrification were adressed by Morfill et al. (1993). However, in two of these processes, liquid water plays a role, so it is questionable whether these processes are relevant. Another approach has been to consider the so-called Elster-Geitel effect, which allows a straightforward calculation and requires a preexisting electrical field. Due to this field the charge carriers of objects in a conducting contact distribute unequally. If the objects are separated, a net charge remains on each. Pilipp et al. (1998) chose the Elster-Geitel effect and another "unspecified" charging process. The Elster-Geitel effect is extremely weak and was therefore already refused by Gibbard et al. (1997), Pilipp et al. (1998), and Poppe et al. (2000b). Furthermore, it is questionable whether a sufficient electrical charge flow can occur beetween nonconducting materials in the short contact time of a collision. Desch \& Cuzzi (2000) used triboelectric series by Harper (1967) based on the elemental composition of grains in the solar nebula. They assumed by far the strongest charge transfer compared to other literature on nebular lightning; as an example, they describe the charging such that a fine metal grain of $1 \mu \mathrm{m}$ diameter colliding with a $600 \mu \mathrm{m}$ diameter silicate object will accept 3000 negative elementary charges independent of collision velocity. Also Sternovsky et al. (2002) used work function differences for explaining experimental results on regolith-simulant charging. With the lack of a satisfying theoretical description, it does not seem surprising that authors of astrophysical literature often avoided to address the nature of tribocharging (Gibbard et al. 1997; Love et al. 1995). Gibbard et al. (1997) assumed an amount of charge transfer extrapolated from an empirical formula by Norville et al. (1991) for the charge transfer between colliding hail and graupel in terrestrial thunderstorms.

\subsection{Summary}

The literature study showed that numerous electrification processes are treated, which are hard to separate, and that the criteria for prediction are vague if available at all. Both negative and positive charge carriers are considered as changing the objects, and at least the material, relative motion, temperature, the details of the separation process, and impurities are among the several causes of charge transfer. As a consequence, we refuse to assume the contact charging hypothesis without further reasoning or to explain tribocharging by a single process. Former astrophysical assumptions about the amount of charge transfer had a wide range over three orders of magnitude, so both the processes and the amount of charge transfer are problems which call for new laboratory measurments.

\section{Experiments}

\subsection{Analogous materials: Dust samples and targets}

The selection of material was lead by the intention of resembling properties of grains in the solar nebula. Hard grains of micron size consisting of elements with high cosmic abundance were chosen, both of spherical and irregular shapes.

Figures 1 and 2 describe the micrometer-sized dust samples by SEM (scanning electron microscope) images and size distributions. Most of the grains had already been used in other experiments, so that further details about the grains and their relevance can be found in Poppe et al. (2000a) and 

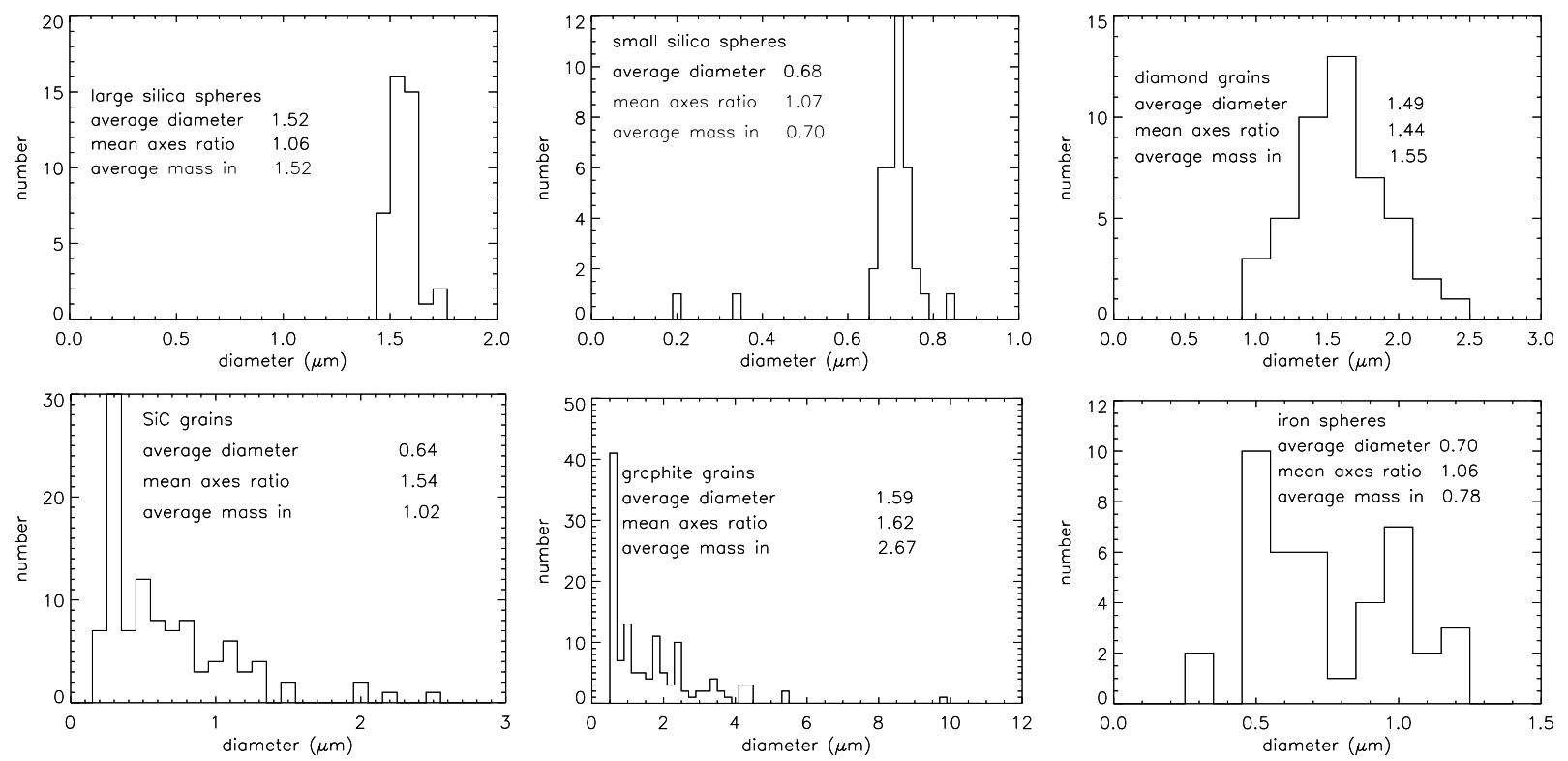

Fig. 2. Size distributions with average diameter, mean axes ratio, and the size of a particle with average mass.

Poppe (2003). The size distribution was determined from SEM images and needs some explanation. The "diameter" $d_{i}$ of particle $i$ is the mean of long and short axes presented on the SEM images. The average diameter is the mean $d$ of $n$ particles. The nominal diameters $d_{n}$, which were assigned above to the particles, represent the diameter of a particle close to average mass $\left.\left(d_{n}=\frac{1}{n}\left(\sum_{i=1}^{n} d_{i}^{3}\right)^{\frac{1}{3}}\right)\right)$. The nominal diameter $d_{n}$ is not only a measured value but also defines a characteristic example particle of each powder sample, which has the mass of a spherical particle with $d_{n}$ and to which a certain charge transfer given in elementary charges is later related.

The targets were plates ( $53 \mathrm{~mm}$ by $38 \mathrm{~mm}$ ) of aluminum, pure iron, and graphite. This choice was guided not only by astrophysical relevance but also by the necessity to use conductive materials. The targets were cleaned by being wiped with paper, purged with ethylene, and subsequently dried by pressurized air.

\subsection{Experimental procedure}

We created particle jets in a vacuum chamber by pushing a dust sample onto the front side of a rotating cogwheel as described in Poppe et al. (1997) and as shown in Fig. 3. This creates jets of single particles which tangentially leave the cogwheel and which we directed onto electrically-conducting targets. For a single experimental run, a piston was pushed with a feed of $1 \mathrm{~mm}$ through the dust storage hole. According to Fig. 3, the target and the cogwheel (diameter $200 \mathrm{~mm}$, width $5 \mathrm{~mm}$ ) were electrically connected to separate channels of an oscilloscope such that we had an independent charge separation measurement both at the cogwheel and at the target. The charge flow is typically on the order of $Q=10^{-7} \mathrm{C}$ for a feed of $1 \mathrm{~mm}$. The oscilloscope indicated the values of $\int U_{\mathrm{C}}(t) \mathrm{d} t=R Q_{\mathrm{C}}$ and $\int U_{\mathrm{T}}(t) \mathrm{d} t=R Q_{\mathrm{T}}$ from which the charge separation at the target $Q_{\mathrm{T}}$ and at the cogwheel $Q_{\mathrm{C}}$ can be calculated. Typically

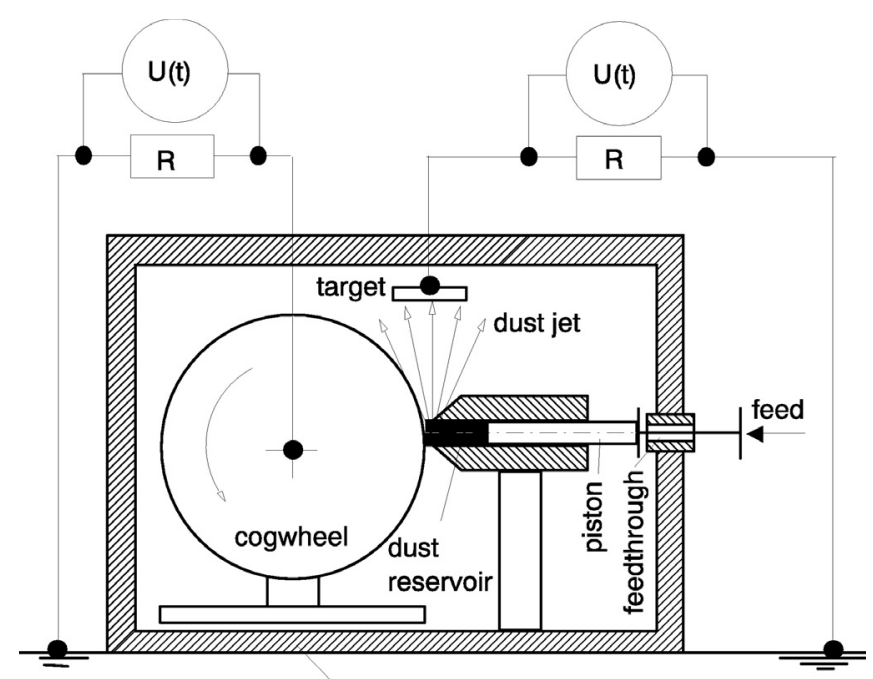

Fig. 3. Schematics of the experimental setup.

10-20 of these values were taken for each combination of particle sample and target in order to prove reproducibility. The air pressure in the experimental setup was normally 0.006 mbar, it never exceeded 0.008 mbar. Experiments took place at room temperature.

The particle mass in the dust storage hole was determined by weighing. With $Q_{\mathrm{C}}$ measured, the charge separation at the cogwheel per powder mass dispersed was calculated. The charge separation at the cogwheel and the dust mass dispersed are correlated within a few percent. This was obvious from the appearance of the $U(t)$ functions of target and of cogwheel which are displayed by the oscilloscope and which show a proportionality. A further indication for this is the scatter of the data points in Fig. 6, which is presented in Sect. 3.4. For a given condition, $Q_{\mathrm{T}} / Q_{\mathrm{C}}$ varies with $\approx \pm 5 \%$. (As an exception to be explained in Sect. 3.3, the correlation does not occur with iron grains.) With this calibration, $Q_{\mathrm{C}}$ allowed us to calculate 
Table 1. Average number of elementary charges given to the targets made of aluminum ( $\mathrm{Al}$ ), iron (Fe), or graphite (C) by a particle of nominal size $d_{n}$ (i.e. positive sign means that the target charged positively, the particle negatively). The precharge is the number of elementary charges given on average to the cogwheel. Error is $\pm 13 \%$.

\begin{tabular}{ccccccccc}
\hline \hline Code & Material & $\begin{array}{c}d_{n} \\
\mu \mathrm{m}\end{array}$ & $\begin{array}{c}\text { Particle mass } \\
\mathrm{kg}\end{array}$ & $\begin{array}{c}\text { Velocity } \\
\mathrm{m} \mathrm{s}^{-1}\end{array}$ & Precharge & $\mathrm{Al}$ & $\mathrm{Fe}$ & $\mathrm{C}$ \\
\hline $\mathrm{a}$ & $\mathrm{SiO}_{2}$ & 0.70 & $3.6 \times 10^{-16}$ & 49 & 61 & 130 & 118 & 35 \\
$\mathrm{~b}$ & $\mathrm{SiO}_{2}$ & 0.70 & $3.6 \times 10^{-16}$ & 69 & 161 & 432 & 301 & 32 \\
$\mathrm{c}$ & $\mathrm{SiO}_{2}$ & 1.52 & $3.7 \times 10^{-15}$ & 59 & 601 & 1561 & 1254 & 125 \\
$\mathrm{~d}$ & $\mathrm{SiO}_{2}$ & 1.52 & $3.7 \times 10^{-15}$ & 106 & 1557 & 3058 & 3166 & -54 \\
$\mathrm{e}$ & diamond & 1.55 & $6.1 \times 10^{-15}$ & 38 & 1335 & 2939 & 1941 & 2673 \\
$\mathrm{f}$ & diamond & 1.55 & $6.1 \times 10^{-15}$ & 67 & 1585 & 3378 & 2085 & 2763 \\
$\mathrm{~g}$ & graphite & 1.44 & $3.4 \times 10^{-15}$ & 38 & 826 & 2456 & 1821 & 415 \\
$\mathrm{~h}$ & graphite & 1.44 & $3.4 \times 10^{-15}$ & 67 & 1022 & 3335 & 1842 & 62 \\
$\mathrm{i}$ & $\mathrm{SiC}$ & 1.02 & $1.7 \times 10^{-15}$ & 38 & 128 & 318 & 382 & 340 \\
$\mathrm{j}$ & $\mathrm{SiC}$ & 1.02 & $1.7 \times 10^{-15}$ & 67 & 219 & 378 & 393 & 375 \\
\hline
\end{tabular}

the powder mass dispersed in an individual experimental run within $\pm 5 \%$.

Because some particles passed beside the target, the percentage of particles hitting the target was determined by weighing a greased target, to which all hitting particles stuck, before and after dust flux exposure. Several repeated measurements with weighing the dust reservoir and the greased target both before and after an experimental run showed that, depending on the dust sample, between 36 and 45 percent of the grains dispersed hit the target and that the standard deviation is $8 \%$.

Two cogwheel circumference velocities, 38 and $67 \mathrm{~m} \mathrm{~s}^{-1}$, were applied. The velocity distribution of the $\mathrm{SiO}_{2}$ spheres was measured by imaging the particle trajectory using the forward scattered light of a modulated laser diode as described in Poppe et al. (2002, 2000a). This method was not applicable for any of the other dust samples because the scattered light was too faint. As a consequence, we had to find a best estimate for the average particle velocity which should mainly lie between the circumference velocity of the cogwheel (fully inelastic collision) or twice this value (fully elastic collision). According to Poppe et al. (2000a), irregularly-shaped grains tend to almost fully inelastic collisions. We therefore assume that, after contact with the cogwheel, the particle speed equals the circumference velocity.

\subsection{Results with varied material, grain size, and velocity}

The average charge separation per particle was calculated using $Q_{\mathrm{T}}$ and the particle mass and percentage hitting the target according to Sect. 3.2. As said in that section, this procedure is not feasible for the iron spheres, which will therefore be treated separately below. The results of all other samples are shown in Table 1 . The errors correlating $Q_{\mathrm{C}}$ and the dust mass dispersed $( \pm 5 \%)$, as well as the error of the percentage of dust hitting the target ( $\pm 8 \%$, Sect. 3.2), add up to a total error of $\pm 13 \%$. Particles were precharged before target collision due to contact with the cogwheel, and therefore, also the average number of elementary charges given to the cogwheel dust disperser is listed.
We find: (1) in general, particles charge negatively when hitting targets and when hitting the dust disperser; (2) micron-sized grains with collision velocities around $100 \mathrm{~m} \mathrm{~s}^{-1}$ normally acquire something in the order of hundreds or thousands of negative elementary charges when hitting the target; (3) the generally equal sign of charging at cogwheel and at target shows that precharged particles do not discharge or share their charge with the target but acquire additional charge upon collision. This means the contact is not characterized by conductance, which would allow the precharge to flow to the target. Obviously, electrical charges are localized at the spot where the particle was hit by the cogwheel, which is in general not the same spot where it hits the target; (4) in order to find a functional dependence of collisional tribocharging, the average grain charging according to Table 1 are shown over the kinetic particle energy (Fig. 4). For comparison, results of Poppe et al. (2000b), who investigated individual charging events of $\mathrm{SiO}_{2}$ spheres with $0.5 \mu \mathrm{m}$ and $1.2 \mu \mathrm{m}$ diameter hitting silicon and silica targets, are indicated as well. Poppe et al. (2000b) gave the empirical relation

$n \approx\left(E / 10^{-15} \mathrm{~J}\right)^{p}$

where $n$ denotes the number of elementary charges and $E$ the kinetic energy. The slope $p$ was found to equal 0.83 . Since we had the best-defined experimental conditions (narrow size distribution and uniform spherical shape) with the $\mathrm{SiO}_{2}$ grains, we used the results of collisions with the iron and the aluminum target to calculate a corresponding slope for our new data obtained at higher energies and with other target materials. We find $p=0.79$, which is very similar; (5) only two particle-target combinations exhibit an exceptionally weak charging (less than $1 / 3$ of that one of the $\mathrm{SiO}_{2}$ spheres) of non-uniform sign (particles: graphite grains, $\mathrm{SiO}_{2}$; target: graphite). All other 12 combinations of this work (particles: $\mathrm{SiO}_{2}$, diamond, $\mathrm{SiC}$, graphite; targets: aluminum, iron, graphite) and a further three combinations of former work (particles: $\mathrm{SiO}_{2}$; target: $\mathrm{SiO}_{2}$, Silicon; Poppe et al. 2000b) are to be described as in Eqs. (1), (2), and (4).

Figure 5 shows $Q_{\mathrm{T}}$ and $Q_{\mathrm{C}}$ measured in experimental runs with iron spheres. Using iron spheres, the dust reservoir was electrically insulated. With $1 \mathrm{~mm}$ feed and a measured fraction 


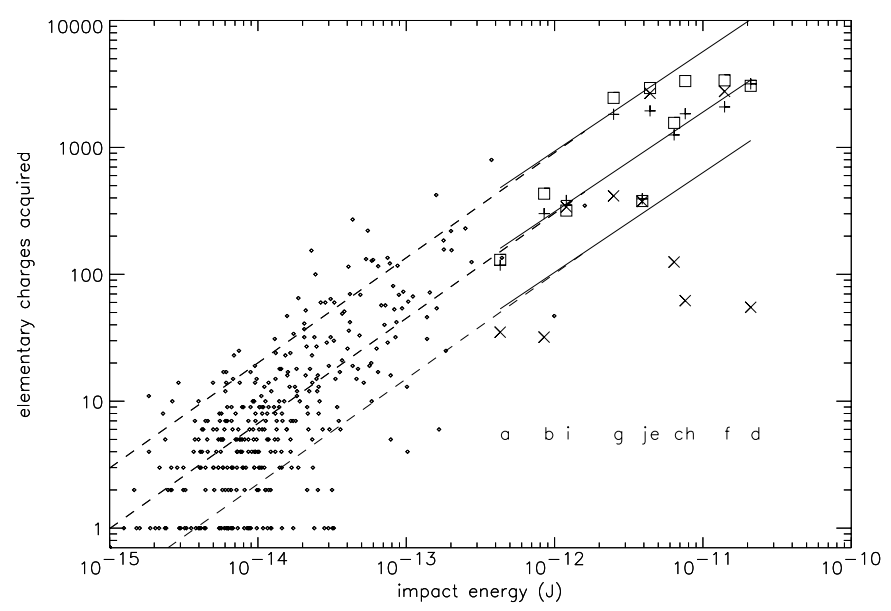

Fig. 4. Comparison of former measurements on charging of individual $\mathrm{SiO}_{2}$ spheres colliding with quartz targets or a silicon wafer (Poppe et al. 2000b, small symbols) and the new measurements of the average charge transfer to conducting targets. The meaning of the codes $a-j$ is the same as in Table 1, and their position on the abscissa shows where to find the corresponding data points. The symbols indicate the target type. Square: aluminium, cross: iron, $\times$-cross: graphite. The center interrupted line represents Eq. (1), the center solid line the one derived from the new data a, b, c, and $d$. The thin outer lines are the center lines times 3 and $1 / 3$.

of $44 \%$ hitting the target, a best estimate is that $2.4 \times 10^{-5} \mathrm{~kg}$ iron spheres hit the target. A particle mass of $1.9 \times 10^{-15} \mathrm{~kg}$ then means that the maximum absolute value of $Q_{\mathrm{T}}=10^{-6} \mathrm{C}$ corresponds to a separation of 510 elementary charges per particle. This is the same order of magnitude as found with most other particles. However, the $Q_{\mathrm{C}}$ values seem to scatter arbitrarily. There is no proportionality between $Q_{\mathrm{T}}$ and $Q_{\mathrm{C}}$; instead often almost no charging occurs, and if any correlation of $Q_{\mathrm{T}}$ with $Q_{\mathrm{C}}$ can be seen, then it is in some cases instead that $Q_{\mathrm{T}}$ decreases with $Q_{\mathrm{C}}$, i.e. the higher the particle was precharged the more of the precharge is given to the target.

\subsection{Results with target temperature variation}

To explore the influence of a temperature gradient between colliding objects, we connected a thermocouple to the target. We then heated the target by a hot air stream, evacuated the chamber, and did measurements as described in Sect. 3.2 while the target slowly cooled down to room temperature. Thus measurement data at numerous temperature differences between target and particle could be obtained. Corresponding experiments were made after cooling the target with a piece of dry ice before evacuation.

Figure 6 shows the charge ratio $\frac{Q_{\mathrm{T}}}{Q_{\mathrm{C}}}$ as a function of target temperature while the powder sample was at room temperature. Since the particle mass dispersed and $Q_{\mathrm{C}}$ are correlated, the charge ratio $\frac{Q_{\mathrm{T}}}{Q_{\mathrm{C}}}$ over target temperature is a relative measure for the charge transfer in a single particle collision at the target. The absolute value of charge transfer at room temperature can be seen from Sect. 3.3. Data are available for $\mathrm{SiO}_{2}$ spheres colliding with an iron and with an aluminum target and for diamond grains hitting an aluminum target. With all material
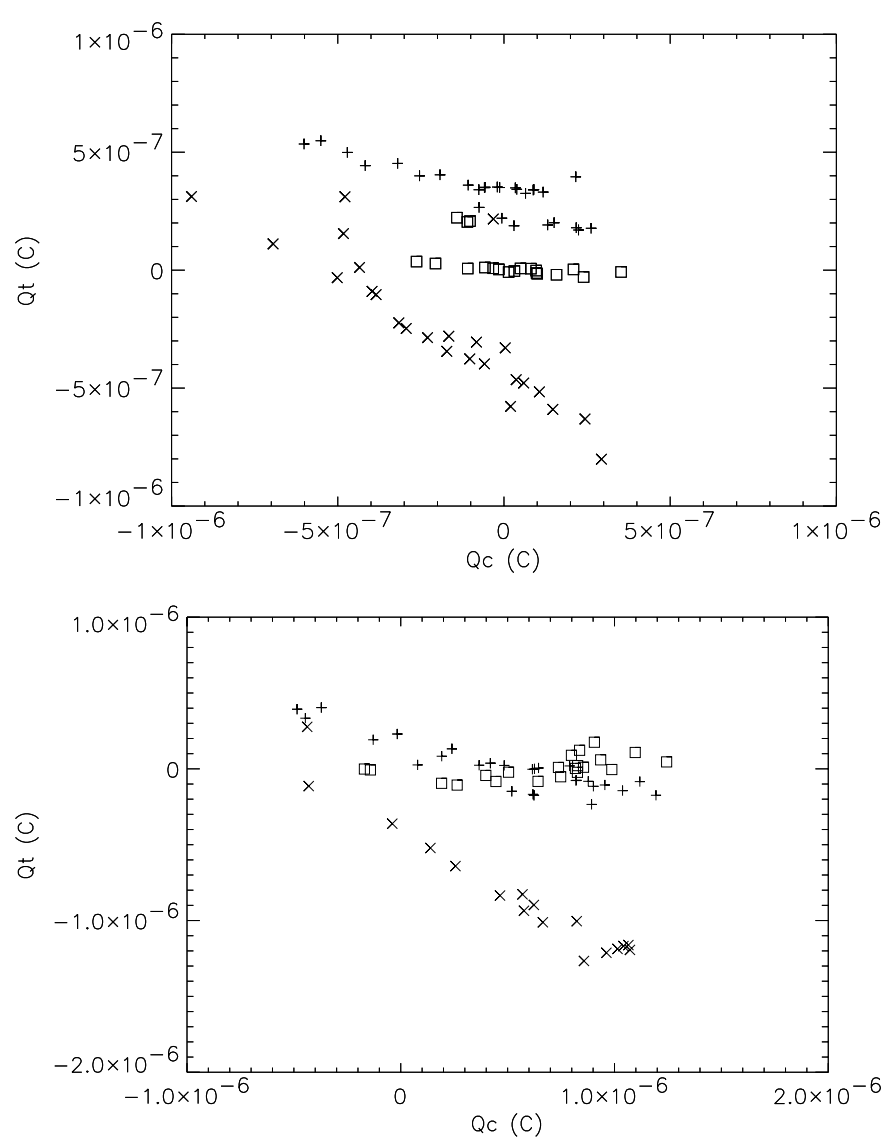

Fig. 5. The measured charge separations with iron spheres at cogwheel $\left(Q_{\mathrm{C}}\right)$ and targets $\left(Q_{\mathrm{T}}\right)$ for a given feed of $1 \mathrm{~mm}$. On top with a cogwheel velocity of $38 \mathrm{~m} \mathrm{~s}^{-1}$, on the bottom with $67 \mathrm{~m} \mathrm{~s}^{-1}$. Square: aluminum target. Cross: iron target. $\times$-cross: graphite target.

combinations, a target temperature rising above particle temperature increased charge transfer. Reducing the target temperature below particle temperature at first reduced the charge transfer to a minimum, and further cooling then led again to an increase in the charge transfer. While this qualitative description is true for all particle-target combinations, there are quantitative differences. The target temperature changed the charging of $\mathrm{SiO}_{2}$ spheres by up to $\pm 50 \%$ while this is in the $10 \%$ range for diamond grains. Also, the minimum charging occurs with diamonds at target temperatures $5-10 \mathrm{~K}$ below the particle temperature whereas this is $24 \mathrm{~K}$ for the silica spheres. Whether an iron or aluminum target is hit by $\mathrm{SiO}_{2}$ spheres makes minor differences, if any.

\section{Discussion}

\subsection{Tribocharging processes}

Temperature differences between colliding objects turned out to play a major role in influencing the charge transfer by up to a factor of three and to be effective with all three material combinations tested. We also see a major influence of the collision energy, i.e. grain mass and velocity are important as already found before with lower collision velocities (Poppe et al. 2000b). This suggests that both effects are linked if we assume a collisioninduced temperature gradient which results from hardness 


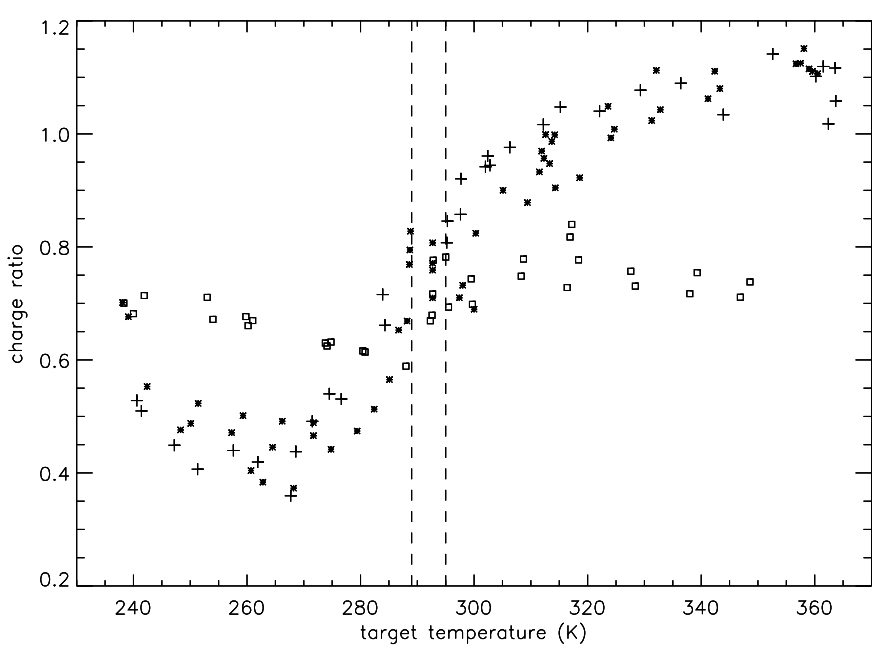

Fig. 6. The charge ratio $\frac{Q_{\mathrm{T}}}{Q_{\mathrm{C}}}$ as a function of target temperature. The vertical lines mark the range of particle temperature (room temperature). Cross: iron target and $\mathrm{SiO}_{2}$ spheres. $\times$-cross: aluminum target and $\mathrm{SiO}_{2}$ spheres. Square: aluminum target and diamond grains. All experimental runs were carried out at $67 \mathrm{~m} \mathrm{~s}^{-1}$ circumference velocity of the cogwheel dust disperser.

differences of the materials and the mainly plastic character of the collision. The assumption is based on the fact that generally the target deforms because all particle materials except the graphite grains are harder than the targets (Lautz \& Taubert 1968 , p. 23). Furthermore, this deformation should mainly be plastic, as measurements on the kinetic energy loss of colliding micrometer-sized grains indicated which were partially carried out with the same particle samples (Poppe et al. 2000a). As known from the experiments with heated targets, a target temperature increase favors the transfer of negative charges to the particle. Both the temperature and the energy dependence of charge transfer are in agreement with the hypothesis that negative charge carriers move by thermal diffusion.

An upper limit for the collision-induced temperature gradient can be estimated by assuming that a volume plastically deforms at the yield strength $\sigma$ and that the collision energy is converted into thermal energy. The volume deformed is $V=\frac{E}{\sigma}$, it heats up by $\Delta T=\frac{E}{V \rho c}$ with $\rho$ the density and $c$ the heat capacity. For pure iron, we find $\Delta T=\frac{\sigma}{\rho c}=33 \mathrm{~K}$ with $\sigma=$ $1.2 \times 10^{8} \mathrm{~Pa}$ (manufacturer's specification), $\rho=7.8 \times 10^{3} \frac{\mathrm{kg}}{\mathrm{m}^{3}}$, and $c=470 \frac{\mathrm{J}}{\mathrm{kg} \mathrm{K}}$. In reality, the heat will be conducted into the target or to the particle, which may reduce $\Delta T$ at the contact surface, and cooling should be the more effective the smaller the volume $V$ is, i.e. the smaller $E$ is. If the temperature gradient vanishes, the charge transfer should be minimal. Regarding the experiments with best defined conditions $\left(\mathrm{SiO}_{2}\right.$ and iron or aluminum target), the target temperature of minimum charge transfer is $24 \mathrm{~K}$ below the particle temperature, a value below the upper limit of $\Delta T$. According to Lowell and Rose-Innes (1980), a small temperature difference is necessary for significant charging. They describe an example of thermally diffusive ion transfer in which a gradient of only $1 \mathrm{~K}$ results in a surface charge density of $10^{-5} \mathrm{C} \mathrm{m}^{-2}$. Graphite targets are soft, which means the effect as described would be weak. Then the heat distributes over a large volume and the temperature increase $\Delta T$, the driving force, is small. In fact, graphite targets charged weakly with most grains. Hence, a temperature gradient caused by initial temperature or by collision-induced energy dissipation in the softer of the colliding objects explains many observations. A temperature gradient as driving force is consistent with the idea of thermally diffusing charge carriers as treated by Lowell \& Rose-Innes (1980), Bowles (1961) and Harper (1967). In our case with generally negative particle charging, thermal diffusion would primarily involve negative charge carriers.

Thermal diffusion of negative charge carriers does not explain our results completely. Undoubtedly, there is one or more competing processes of tribocharging besides the most important one, a finding which should not be surprising in light of the numerous tribocharging processes (Sect. 2). We see this from the results with the cooled target which, at a certain temperature, should compensate for any collision-induced temperature increase, thus, with the temperature difference of zero, the driving force of thermal diffusion vanishes. Nevertheless, a reduced charge transfer remained, suggesting that there was more than one charge transfer process. Further cooling increases the charge transfer again which, in the frame of thermal diffusion, must be explained with positive charge carriers moving from the particle to the target. The consideration of positive charge carriers should keep us from restricting our interpretation of charge transfer on electron transfer. If positive ions apparently play a role and were previously found to play a role in thermally diffusive charge transfer (Bowles 1961) there is per se no reason to refuse the idea that tribocharging may also involve negative ions. Furthermore, there are additional observations which do not fit in the frame of thermal diffusion and make us aware that other tribocharging processes are effective. Graphite grains are softer than the metallic targets and so, one would expect them to deform and heat up, resulting in the opposite sign of charging compared to the hard grains which instead deform the target. However, this is not the case. Also, thermally-diffusive charge transfer cannot explain why diamond and $\mathrm{SiC}$ grains accepted so many negative charges when hitting the graphite target, whereas the other grains charged weakly with non-uniform sign.

The maximum charge transfer to the iron spheres is comparable with the one found with other particles. However, their further behavior is different and marked by their conductivity. In contrast to other particles, $Q_{\mathrm{C}}$ and thus their precharge, varies, which could be caused by a reflow of charges during the separation from the dust reservoir and from the cogwheel. Such a reflow could then reduce electrification to a certain extent. Also, conducting particles can give their precharge to the target, as can be seen from the results with the graphite target, where we often find decreasing $Q_{\mathrm{T}}$ with increasing $Q_{\mathrm{C}}$.

We rule out material-dependent contact charging as the dominant process of tribocharging in our experiments for several reasons: if it had been dominant, the choice of material would have been the major influence on charge transfer, which was not true. Also, like materials get charged at a strength comparable to other combinations, which cannot be explained by material-dependent contact charging. This is true based on 
elemental compositions such as diamond grains and graphite targets or even on molecular compositions, as found by Poppe et al. (2000b) using $\mathrm{SiO}_{2}$ both as particle sample and as target. Finally, triboelectric series require the sign of charging to depend on the material combination. According to the series used by Desch \& Cuzzi (2000), silicates would charge positively when hitting iron, while they charged negatively in our experiments. Refusing material-dependent contact charging does not mean that we deny the existence of contact charging, not even that it does not play a role as a secondary process in our experiments. However, material-dependent contact charging is not of major importance in our experimental conditions.

\subsection{Application recipe for astrophysical models}

Independent of the physical details of tribocharging, there is a need for assumptions on the amount of collisional charge transfer between solids in the solar nebula or in comparable environments. For future applications, we propose Eq. (1) with $p=0.8$, if collision energies $E$ in single grain collisions are below $2 \times 10^{-11} \mathrm{~J}$ and conductivity is so low that there is no significant reflow of charges during the separation process. The latter criterion is always fulfilled if one object is an insulator. If two metals collide, grain electrification is in the same order of magnitude as for other materials; however, details of the separation process become important, which can lead to a reflow of charge accumulations (Lowell \& Rose-Innes 1980) and which cannot be treated in this article. Accuracy of the prediction is within a factor of three, for which only a few exceptions were found (Sect. 3.3). Concerning grain size, grain material, and collision velocities, our experimental parameters are relevant for preplanetary dust aggregation (see Sect. 1). On the other hand, the preplanetary grain growth is expected to have lead to porous aggregates. Consequently, the charge transfer of aggregates colliding with single grains or other aggregates is an important case. Although physical details of aggregate collisions have been investigated experimentally (e.g. Wurm \& Blum 1998, 2000), there are no measurements of triboelectrification in collisions of such aggregates. On a macroscopic scale, aggregates have other mechanical and thermal properties than single grains, and so the question arises as to what extent our results can be transferred to aggregate collisions. Since there is no reason why the dominating temperature-driven mechanism of charge transfer should work fundamentally different if a micron-sized particle hits an equally-sized particle or a larger object, such as our cm-sized targets, the charge transfer of particles that constitute an aggregate can be estimated from our results. In the velocity regime investigated here (38 to $106 \mathrm{~m} \mathrm{~s}^{-1}$ ), aggregates are expected to fragment in their constituent particles (Wurm \& Blum 2000), and a charge transfer will then occur according to the number and energy of all single grain collisions.

\subsection{Astrophysical problems in the light of the results}

The preplanetary dust aggregation process requires a relative motion between solids, collisions, and sticking. The particle relative motion has been addressed as a result of forces only caused by the dilute gas of the solar nebula (Weidenschilling \& Cuzzi 1993), and such forces and motions were investigated in detail (Wurm \& Blum 1998; Blum et al. 2000). In the light of tribocharging experiments, Poppe et al. (2000b) pointed out the possiblity that interactions with a magnetic field could exert larger forces on the particles than the gas does. We now find even higher grain charging for higher collision velocities, and therefore the question whether this restriction to aerodynamic forces is adequate seems even more justified but can, on the other hand, not be answered by experiments on tribocharging alone.

Also the sticking efficiency is an important parameter for the dust aggregation. Experimental studies by Poppe et al. (2000a) showed a considerably higher sticking probability than thought before according to theoretical work by Chokshi et al. (1993). It has also already been shown in experiments that tribocharging can contribute to the sticking efficiency (Poppe et al. 2000b, Sect. 1). This can happen if a particle bounces off and is subsequently trapped in a field of the target which was electrified by preceeding collisions. While so far particle trapping was only observed for collision velocities below $20 \mathrm{~m} \mathrm{~s}^{-1}$ and only charge transfer measurements in this range were available, our experiments showed a high and energy-dependent tribocharging up to $100 \mathrm{~m} \mathrm{~s}^{-1}$, i.e. over a wide range of collision velocities expected for the solar nebula, which thus stresses the importance of tribocharging for favoring the preplanetary dust aggregation process.

However, whether electrostatic trapping happened or not depended on the individual collision conditions, i.e. on the amount and sign of collisional charge transfer, on the charge carried by the target due to preceeding collisions or other charging or discharging effects, and on the energy loss in the bouncing collision. This means, that non-sticking collisions should have existed as well, and that the charge transfer in such collisions is a key process for the possibility of lightning (Sect. 1). As an example, for an iron grain colliding with a silicate grain, Desch \& Cuzzi (2000) assumed a charge separation of 3000 elementary charges (Sect. 2). According to Sect. 3.3, we expect that an iron grain of this size $\left(m=4.1 \times 10^{-15} \mathrm{~kg}\right)$ will carry 200,900 , or 2800 negative charges in the case of 20 , 50 , or $100 \mathrm{~m} \mathrm{~s}^{-1}$ collision speed, respectively. Compared to all work on nebular lightning cited in the introduction, the one by Desch \& Cuzzi (2000) was based on modelling assumptions on the amount of charge transfer, which are closer to our results than the assumptions of other authors. It therefore seems that nebular lightning was not hampered by a too low charge exchange in particle collisions because Desch \& Cuzzi (2000) concluded that lightning over large regions $(\approx 100 \mathrm{~km})$ could have been both possible and sufficiently energetic to have formed chondrules.

\section{Conclusions}

We presented charge transfer measurements in grain-target collisions under conditions relevant to the solar nebula. Our results enlarged the experimental data base such that higher collision energies, more material combinations including 
conductive solids, and temperature differences among colliding objects were investigated than before. The charge transfer was a function of the collision energy and of the temperature difference of the colliding objects. The most plausible explanation for these and other results is that thermal diffusion of negative charge carriers was the most important, but not the only, charge transfer process, whereas material-dependent contact charging which is a very often discussed hypothesis on tribocharging was not the dominant process. Compared to former assumptions in astrophysical literature, the charge transfer is high thus stressing the importance of tribocharging in solid grain collisions in the solar nebula.

\section{References}

Blum, J., Wurm, G., Kempf, S., et al. 2000, Phys. Rev. Lett., 85, 2426 Bowles, A. H. 1961, The effect of a Thermal Gradient upon Charge Separation between Similar Polyethylene Surfaces, Proc. Phys. Soc., 78, 958

Brownlee, D. E. 1985, Ann. Rev. Earth Planet Sci., 13, 147

Cheng, L., \& Soo, S. L. 1970, J. Appl. Physics, 41, 585

Chokshi, A., Tielens, A. G. G. M., \& Hollenbach, D. 1993, ApJ, 407, 806

Cuzzi, J. N., Dobrovolskis, A. R., \& Champney, J. N. 1993, Icarus, 106, 496

Desch, S. J., \& Cuzzi, J. N. 2000, Icarus, 143, 87

Gibbard, S. G., Levy, E. H., \& Morfill, G. E. 1997, On the Possibility of Lightning in the Protosolar Nebula, Icarus, 130, 517

Harper, W. R. 1967, Contact and Frictional Electrification, (Oxford: Clarendon Press)

Hewins, R. H. 1996, Chondrules and the Protoplanetary Disk: An Overview, in Chondrules and the Protoplanetary Disk, ed. R. H. Hewins, R. H. Jones, \& E. R. D. Scott (Cambridge: Cambridge University Press), 3

Jessberger, E. K., Stephan, T., Rost, D., et al. 2001, Properties of interplanetary dust: Information from collected samples, in Interplanetary dust, ed. E. Grün, B. Å. S. Gustafson, S. F. Dermott, $\&$ H. Fechtig (Berlin and Heidelberg: Springer Verlag), 253

John, W., Reischl, G., \& Devor, W. 1980, J. Aerosol Sci., 11, 115
Kacprzyk, R., \& Gajewski, J. 2001, J. Electrostatics, 51/52, 124

Kerridge, J. F. 1993, Icarus, 106, 135

Lautz, G., \& Taubert, R. 1968, Kohlrausch - praktische Physik, Band 3, Tafeln (Stuttgart: B.G. Teubner)

Love, S. G., Keil, K., \& Scott, E. R. D. 1995, Icarus, 115, 97

Lowell, J. 1986, J. Phys. D: Appl. Phys., 19, 105

Lowell, J., \& Rose-Innes, A. C. 1980, Adv. Phys. 29, 947

Masuda, H., Komatsu, T., \& Iinoya, K. 1976, A.I.Ch.E.J., 22, 558

Matsuyama, T., \& Yamamoto, H. 1989, IEEE Transactions on Industry Applications 30, 602

Morfill, G., Spruit, H., \& Levy, E. H. 1993, Physical Processes and Conditions Associated with the Formation of Protoplanetary Disks, Protostars and Planets III, ed. E. Levy, \& J. Lunine (Tucson: University of Arizona Press), 939

Norville, K., Baker, M., \& Latham, J. 1991, J. Geophys. Res., 96, 7463

Pilipp, W., Hartquist, T. W., Morfill, G. E., \& Levy, E. H. 1998, A\&A, 331,121

Poppe, T. 2003, Icarus, 164, 139

Poppe, T., Blum, J., \& Henning, T. 1997, Rev. Sci. Instrum., 68, 2529

Poppe, T., Blum, J., \& Henning, T. 2000a, ApJ, 533, 454

Poppe, T., Blum, J., \& Henning, T. 2000b, ApJ, 533, 472

Poppe, T., Wurm, G., \& Krieg, R. 2002, Measurement Sci. Technol., 13, 796

Prochazka, R. 1966, Staub, 26(5), 202

Rietmeijer, F. J. M. 1993, Earth and Planet. Sci. Lett., 117, 609

Rietmeijer, F. J. M. 2002, Chemie der Erde, 62, 1

Sandford, S. A. 1996, Meteor. Planet. Sci., 31, 446

Schräpler, R., \& Henning, T. 2004, ApJ, 614, 960

Sternovsky, Z., Robertson, S., Sickafoose, A., Colwell, J., \& Horányi, M. 2002, J. Geophys. Res., Planets, 107, 5105

Vercoulen, P. 1995, Electrostatic Processing of Particles, - a Tool in Particle Technology, Thesis Technical University of Delft

Weidenschilling, S. J., \& Cuzzi, J. N. 1993, Formation of Planetesimals in the Solar Nebula, in Protostars and Planets III, ed. E. Levy, J. Lunine, \& M. S. Matthews (Tucson: University of Arizona Press) 1031

Wurm, G., \& Blum, J. 1998, Icarus, 132, 125

Wurm, G., \& Blum, J. 2000, Icarus, 143, 138

Whipple, F. L. 1966, A Suggestion as to the Origin of Chondrules, SAO Special Report 206 